\title{
A Comparative Study of Facial, Retinal, Iris and Sclera Recognition Techniques
}

\author{
Sugandha Agarwal1, Rashmi Dubey2, Sugandh Srivastava3, Prateek Aggarwal ${ }^{4}$ \\ ${ }^{I}$ (Amity University, Noida)
}

\begin{abstract}
The pressures on today's system administrators to have secure systems are ever increasing. One area where security can be improved is in authentication. Face, retina, iris and sclera recognition, biometrics, provide one of the most secure methods of authentication and identification. These technologies are very useful in areas such as information security, physical access security, ATMs and airport security. These technologies are more or less accurate, easy to use, non-intrusive, and difficult to forge and, despite what people may think, are actually quite fast systems once initial enrolment has taken place. However, they do require the co-operation of the subject: need specific hardware and software to operate. These recognition technologies do provide a good method of authentication to replace the current methods of passwords, token cards or PINs and if used in conjunction with something the user knows in a two-factor authentication system then the authentication becomes even stronger.
\end{abstract}

Keywords: Biometrics, Iris, Sclera, Retina and Facial Recognition.

\section{Introduction}

Biometrics are used in a wide array of applications, which makes a precise definition difficult to establish. The most general definition of a biometric is:

"A physiological or behavioral characteristic, which can be used to identify and verify the identity of an individual" There are numerous biometric measures which can be used to help derive an individual's identity. They can be classified into two distinct categories:

Physiological - these are biometrics which are derived from a direct measurement of a part of a human body. The most prominent and successful of these types of measures to date are fingerprints, face recognition, irisscans and hand scans.

Behavioral - extract characteristics based on an action performed by an individual, they are an indirect measure of the characteristic of the human form. The main feature of a behavioral biometric is the use of time as a metric. Established measures include keystroke-scan and speech patterns.

Biometric identification should be an automated process. Manual feature extraction would be both undesirable and time consuming, due to the large amount of data that must be acquired and processed in order to produce a biometric signature. Inability to automatically extract the desired characteristics which would render the process infeasible on realistic size data sets, in a real-world application.

Recently, human recognition techniques have attracted increasing attention in intelligent surveillance applications. For recognition systems using Physiological feature-based human recognition systems use individual, unique characteristics such as finger prints, iris and face. Because of the unique nature, the Physiological feature based system provides very high recognition rate upto $90 \%$. This paper aims at development of a biometric kit which uses the physiological characteristic to identify and verify the identity of an individual.

To the best of our knowledge, in the literature, only a few comparisons have been appeared in the field of computing. In this paper we bring out a complete comparison of the various models used in human recognition.

\section{Recognition Models}

In this section, we briefly explain the various models used in the field of biometrics.

\section{Facial Scan:}

This technology is considered a natural means of biometric identification since the ability to distinguish among individual appearances is possessed by humans. Facial scan systems can range from software-only solutions that process images processed through existing closed-circuit television cameras to full-fledged acquisition and processing systems, including cameras, workstations, and back- end processors. With facial recognition technology, a digital video camera image is used to analyze facial characteristics such as the distance between eyes, mouth or nose. These measurements are stored in a database and used to compare with a subject standing before a camera. Facial recognition systems are usually divided into two primary groups. First there is what is referred to as the 'controlled scene' group whereby the subject being tested is located in a known 
environment with a minimal amount of scene variation. In this case, a user might face the camera, standing about two feet from it. The system locates the user's face and performs matches against the claimed identity or the facial database. The system usually comes to a decision in less than 5 seconds. The other group is known as the "random scene" group where the subject to be tested might appear anywhere within the camera scene. This situation might be encountered within a system attempting to identify the presence of an individual within a group or crowd. Facial-scan technology is based on the standard biometric sequence of image acquisition, image processing, distinctive characteristic location, template creation, and matching. An optimal image is captured through a high-resolution camera, with moderate lighting and users directly facing a camera. After the acquisition the process of image processing takes place. Color images are normally reduced to a black and white and images cropped to emphasize facial characteristics. Images are normalized to account for orientation and distance. Images can be enlarged or reoriented as long as a point between the eyes serves as a point of reference. The processes of characteristic location can then take place. There are several matching methods available for facial scans which attempt to match visible facial features in a fashion similar to the way people recognize one another. Areas of the face not apt to change over time such as sides of the mouth, nose shape and areas around the cheekbones, distinctive characteristics most often used in image matching. Areas likely to change over time, such as ones hairlines are not normally used for verification.

\section{Retina Scan}

Retina-scan technology makes use of the retina, which is the surface on the back of the eye that processes light entering through the pupil. Retinal Scan technology is based on the blood vessel pattern in the retina of the eye. The principle behind the technology is that the blood vessels at the retina provide a unique pattern, which may be used as a tamper-proof personal identifier. Since infrared energy is absorbed faster by blood vessels in the retina than by surrounding tissue, it is used to illuminate the eye retina. Analysis of the enhanced retinal blood vessel image then takes place to find characteristic patterns. Retina-scan devices are used exclusively for physical access applications and are usually used in environments that require high degrees of security such as high-level government military needs. Retina-scan technology was developed in the 1980's, is well known but probably the least deployed of all the biometric technologies. Additionally, retina-scan technology is still in a prototype development stage and still commercially unavailable. Retina-scan technology image acquisition is difficult in that the retina is small and embedded, requiring specific hardware and software. The user positions his eye close to the unit's embedded lens, with the eye socket resting on the sight. In order for a retinal image to be acquired, the user must gaze directly into the lens and remain still; movement defeats the acquisition process requiring another attempt. A low intensity light source is utilized in order to scan the vascular pattern at the retina. This involves a 360 degree circular scan of the area taking over 400 readings in order to establish the blood vessel pattern. This is then reduced to 192 reference points before being distilled into a digitized 96 byte template and stored in memory for subsequent verification purposes. Normally it takes 3 to 5 acceptable images to ensure enrollment. Because of this, the enrollments process can be lengthy. Enrollments can take over 1 minute with some users not being able to be enrolled at all. It seems the more that a user is acclimated to the process, the faster the enrollment process works. After image acquisition, software is used to compile unique features of the retinal blood vessels into a template. Retina-scan technology possesses robust matching capabilities and is usually configured to do one-to-many identification against a database of users, however, this technology requires a high quality image and will not enroll a user unless a good image is acquired. For this reason, there is a moderately high false reject rate due to the inability to provide adequate data to generate a match template.

\section{Iris Scan}

The iris is the coloured ring around the pupil of every human being and like a snowflake, no two are alike. Each is unique in its own way, exhibiting a distinctive pattern. The iris is a muscle that regulates the size of the pupil, controlling the amount of light that enters the eye. Iris cameras perform recognition detection of a person's identity by mathematical analysis of the random patterns that are visible within the iris of an eye from some distance. It combines computer vision, pattern recognition, statistical inference and optics.

Of all the biometric devices and scanners available today, it is generally conceded that iris recognition is the most accurate. Iris recognition is rarely impeded by glasses or contact lenses and can be scanned from $10 \mathrm{~cm}$ to a few meters away. The iris remains stable over time as long as there are no injuries and a single enrolment scan can last a lifetime. Even blind people can use this scan technology since iris recognition technology is iris pattern-dependent not sight dependent.

Iris scanning is an ideal way of biometric identification since the iris is an internal organ that is largely protected by damage and wear by the cornea. This makes it more attractive then fingerprints which can be difficult to recognize after several years of certain types of manual labour. The iris is also mostly flat and controlled by 2 muscles so it helps make the iris movements more predictable then facial recognition.

Iris cameras, in general, take a digital photo of the iris pattern and recreating an encrypted digital template of 
that pattern. That encrypted template cannot be re-engineered or reproduced in any sort of visual image. Iris recognition therefore affords the highest level defence against identity theft, the most rapidly growing crime.

The imaging process involves no lasers or bright lights and authentication is essentially non-contact. Today's commercial iris cameras use infrared light to illuminate the iris without causing harm or discomfort to the subject.

Before scanning of the iris takes place, the iris is located using landmark features. These landmark features, and the distinct shape of the iris allow for imaging, feature isolation and extraction. Localization of the iris is an important step in iris recognition because, if done improperly, resultant noise (i.e.: eyelashes, reflections, pupils and eyelids) in the image may lead to poor performance.

\section{IV.Sclera Scan}

The sclera is the part of the eye commonly known as the "white". It is the outer and protective covering of the eye. It is made up of four layers of tissue i.e. the episclera, stroma, laminafusca and endothelia. It forms the supporting wall of the eyeball, and is continuous with the clear cornea. The blood vessel structure of the sclera is unique to each person, and it can be remotely obtained non intrusively in the visible wavelengths. The structure of blood vessels is visible and stable over time in sclera. With increasing age collasion and elastic fibers deteriorates, sclera dehydration occurs and calcium and lipid salts accumulate but the blood vessels do not deteriorates. Therefore, it is well suited for human identification. Sclera recognition is a challenging research problem because images of sclera vessel patterns are often defocused and/or saturated and, most importantly, the vessel structure in the sclera is multilayered and has complex nonlinear deformations.

Recognition through sclera takes through several stages. Initially after capturing the image of the eye the sclera region of the eye is segmented from the whole. The colored image is converted into greyscale image in order to apply sobel filters which are used to estimate the glare area which is the small bright area of the iris image. Iris boundary is detected using the greyday angular search method. They region of interest i.e. sclera is then segmented. Thresholding is carried put using Otsu's method. The detected sclera is refined by refining the eyelids and the iris and a perfect segmented image is obtained. The segmented sclera image is given to Gabour filter for feature extraction and the resultant is refined with adaptive threshold to make the vessel structure clearly visible and thinning morphological operators are applied to the adaptive thresholded output. These line segments are obtained using line descriptors and are compared with the target template for matching.

\section{Challenges In The Various Recognition Models}

Every recognition model has similar or unique challenges, needs to convert challenges into opportunities and has scope for further research. In this section, we highlight some of the challenges of the various recognition models considered.

\section{Facial Scan}

The research challenges of facial scan model are as follows:

(1) Distance: Challenges that occur in the image acquisition process include distance from user, angled acquisition and lighting. Distance from the camera reduces facial size and thus image resolution.

(2) Position: Users not looking directly at the camera, positioned more than 15 degrees either vertically or horizontally away from ideal positioning are less likely to have images acquired.

(3) Illumination: Lighting conditions, which cause an image to be underexposed or underexposed, can cause challenges.

(4)Skin Tone: Users with a darker skin tone can be difficult to acquire. Select Hispanic, black and Asian individuals can be more difficult to enroll and verify in some facial-scan systems because acquisition devices are not always optimized to acquire darker faces.

\section{Retina Scan}

The research challenges of retina scan model are as follows:

(1) Limited Usage: Challenges include the fact that the technology is difficult to use, users claim discomfort with eye-related technology in general and the fact that retina- scan technology has limited uses.

(2) Expertise: Enrollments require prolonged concentration requiring a well-trained and motivated user. Training the experts accurately can turn out to be time consuming and costly.

(3)Registration Time Retina: Scan enrollments take longer than both iris-scan and fingerprinting. Multiple scans are required at the time of enrollment for precision.

(4) User Interaction: Users claim discomfort with the fact that they must position their eye very close to the device. Users commonly fear that the device itself or the light inside the device can harm their eyes in some way. Many also feel that this retina scans are invasive in that the inability to use the retina can be linked to eye disease. 
(5) Application area: Retina scan has limited uses normally deployed in high security, low volume physical access situations in which inconveniencing users is an acceptable cost of heightened security.

\section{Iris Scan}

IV. The research challenges of iris scan model are as follows:

(1) Procurability: The iris is a very small organ to scan from a distance. It is a moving target and can be obscured by objects such as the eyelid and eyelashes.

(2) Debelity: Subjects who are blind or have cataracts can also pose a challenge to iris recognition, as there is difficulty in reading the iris.

(3)Illumination: The camera used in the process needs to have the correct amount of illumination. Without this, it is very difficult to capture an accurate image of the iris. Along with illumination comes the problem with reflective surfaces within the range of the camera as well as any unusual lighting that may occur. All of these impact the ability of the camera to capture an accurate image.

(4) User Interaction: Although there is minimal intrusiveness with iris recognition, there is still the need for cooperation from subjects to enroll in the system and undergo subsequent authentication scans. Enrolling a noncooperative subject would prove very difficult indeed.

(5)Extensive training: Inadequate training of users at the initial enrolment period will cause problems both at the initial enrolment time and subsequent authentications. Frustrated users will not help make the system any easier to use and will not be accepted by users as a convenient authentication method. Communication with users plays a major part in introducing such a system successfully.

\section{Sclera Scan}

The research challenges of sclera scan model are as follows:

(1) Target Area: Exposed region of sclera is noisy due to the movement of eyelids during image capture.

(2) Computational cost: The heavy computational cost associated with vessel enhancement has been an essential task in order to achieve a reasonably good recognition performance.

(3) Development: Problems are concerned with ways of writing software if we want to integrate this unimodel of recognition with several other existing models. The problems may include decomposing and distributing the processing elements, and then assembling solutions.

\section{Comparison Of The Various Models}

Viewed in a broad sense, the concepts of facial, retina, iris and sclera scan seems to have similar features. This section puts light to differentiate in different perspectives and give an end-to-end comparison. It could be understood easily when represented in a tabular form as given below.

\begin{tabular}{|l|l|l|l|l|}
\hline & $\begin{array}{l}\text { FACIAL } \\
\text { SCAN }\end{array}$ & $\begin{array}{l}\text { RETINA } \\
\text { SCAN }\end{array}$ & IRIS SCAN & SCLERA SCAN \\
\hline Target Area & High & Low & Low & Medium \\
\hline Accuracy & $\begin{array}{l}\text { More than } \\
\text { retina scan }\end{array}$ & Lowest & Low & High \\
\hline Invasive & No & Yes & No & No \\
\hline $\begin{array}{l}\text { Affect of } \\
\text { age }\end{array}$ & Yes & Yes & No & No \\
\hline $\begin{array}{l}\text { User } \\
\text { Interaction }\end{array}$ & Not Required & Required & Required & Requied \\
\hline $\begin{array}{l}\text { Training } \\
\text { Time }\end{array}$ & Average & Time Consuming & Average & Average \\
\hline
\end{tabular}

\section{A. Facial Scan}

\section{Applications Of The Various Recognition Models}

(1) Identification Systems: It can be used as an identification task, where any new applicant being enrolled can be compared against the entire database of previously enrolled claimants, to ensure that he is not claiming under more than one identity.

(2) Surveillance: The application domain where most interest in face recognition is being shown is probably surveillance. Video is the medium of choice for surveillance because of the richness and type of information that it contains and naturally, for applications that require identification, face recognition is the best biometric for video data.

(3) Pervasive Computing: Another domain where face recognition is expected to become very important, although it is not yet commercially feasible, is in the area of pervasive or ubiquitous computing. Many people are envisaging the pervasive deployment of information devices. 


\section{B. Retina Scan}

(1) Security: Retinal recognition has primarily been used in combination with access control systems at high security facilities. This includes military installations, nuclear facilities, and laboratories.

(2) Fraud detection: One of the best-documented applications of retina scan, which is currently being used by the State of Illinois, reduces welfare fraud by identifying welfare recipients thus preventing multiple benefit payments.

(3) Access Control: Since PC cameras have become widespread, their use for retina-based PC logon has become feasible, though take-up seems to be very limited. Increased ease-of-use over password protection is hard to argue with today's somewhat unreliable and unpredictable systems, and for few domains is there motivation to progress beyond the combinations of password and physical security that protect most enterprise computers.

\section{Iris Scan}

(1) Biometric key cryptography: Software based cryptography uses encryption key which are long bit strings. They are very hard to memorize and it can be easily attacked by brute search or technique. Iris uniquely identifies a person and is secure method for generating stream cipher.

(2)IrisFarm: A networked distributed server and communications architecture which allows simultaneous enrollments into the central database without interrupting parallel search queries from multiple distributed stations used in immigration purposes and border crossing applications.

\section{Sclera Scan}

(1) Eyeprint: Like fingerprints every individual have a different eye print reflected by the blood vessels in the sclera of the eye. Even two twins don't have the same eye print.

(2) Gaze tracking: It is the complex process of detecting where a person is looking. Sclera can be used in the development of a gaze tracking system that estimates the eye gaze by using a stable reference point.

(3)Access mechanism for visually impaired- As sclera isn't dependent on eye power, it can successfully be used as a recognition model for people without eyesight.

\section{Conclusion}

In this paper, we have presented a detailed comparison on the various human identification models i.e. face, retina, iris and sclera. The issues and challenges related to these recognition models are highlighted. The projects and applications in various fields are briefly discussed. Also the tools and simulation environments useful for development of applications are highlighted. Such a comparison in different perspectives will make easy to understand the computing models since the features of these computing models seems to be similar conceptually. It also helps in identifying the similarities and differences from each other. Iris and sclera appear to be a promising model especially focusing on standardizing APIs, security, interoperability, and dynamic application for complex services. Hence there is a scope for further research in these areas.

\section{References}

[1] R. Derakhshani,A.Ross,S.Crihalmeanu,A new biometric modality based on conjunctival vasculature, in: Proceedings of Artificial Neural Networks in Engineering (ANNIE2006),St.Louis,Missouri,USA,2006.

[2] S. Crihalmeanu,A.Ross,R.Derakhshani, Enhancement and registration schemes for matching conjunctival vasculature, in:Proceedingsofthe3rd IAPR/IEEE International Conferenceon Biometrics(ICB2009),Italy,2009, pp. 1240-1249.

[3] N.L. Thomas, Y. Du, Z. Zhou, A new approach for sclera vein recognition, in: Proceedings of the International Society for Optical Engineering (SPIE), vol. 7708, 2010.

[4] Z. Zhou,E.Y.Du,N.L.Thomas, A comprehensive sclera image quality measure, in: proceedings of the $11^{\text {th }}$ International Conference on Control, Automation, Robotics and Vision(ICARCV2010),Singapore,2010,pp.638-643.

[5] S.Crihalmeanu,A.Ross,Multispectral scleral patterns for ocular biometric recognition, PatternRecognit.Lett.33(14)(2012)18601869.

[6] R. Derakhshani,A.Ross,Atexture-based neural network classifier for biometric identification using ocular surface vasculature ,in: Proceedings of International Joint Conference on Neural Networks(IJCNN2007),Kensas,USA, 2007,pp.2982-2987.

[7] K.Oh,K.-A.Toh,Extractingsclerafeaturesforcancelableidentityverification,in: Proceedingsofthe5thIAPRInternationalConferenceonBiometrics (ICB 2012),NewDelhi,India,2012.

[8 ] U.Park,R.R.Jillela,A.Ross,A.K.Jain, Periocular biometrics in the visible spectrum, IEEETrans.Inf.ForensicsSecur.6(1)(2011)96106.

[9] S. Bharadwaj,H.S.Bhatt,M.Vatsa,R.Singh, Periocular biometrics:wheniris recognition fails,in:Proceedings of the 4th IEEE International Conference on Biometrics: Theory Applications and Systems(BTAS2010),2010.

[10] P.E.Miller,A.W.Rawls,S.J.Pundlik,D.L.Woodard,Personal $\quad$ identification using $\quad$ periocular $\quad$ skin texture,in:Proceedingsofthe2010ACMSymposiumon Applied Computing,2010,pp.1496-1500.

[11] Daugman JG. High confidence visual recognition of persons by a test of statistical independence. IEEE Trans Pattern Anal Machine Intell 1993;15: 1148-61.

[12] Daugman JG. How iris recognition works. IEEE Trans Circuits Syst Video Technol 2003;14:1-17.

[13] Daugman JG. The importance of being random: statistical principles of iris recognition. Pattern Recognition 2003;36:279-91

[14] A. Haro, I. Essa, M. Flickner, Detecting and tracking eyes by using their physiological properties, in: Proceedings of the Conference on Computer Vision and Pattern Recognition, June 2000.

[15] X. Xie, R. Sudhakar, H. Zhuang. Real-time eye feature tracking from a video image sequence using Kalman Elter, IEEE Trans. 
Syst. Man Cybern. 25 (1995) 1568-1577.

[16] S. Sirohey, A. Rosenfeld, Z. Duric, A method of detecting and tracking irises and eyelids in video, Pattern Recognition 35 (2002) 1389-1401.

[17] Green JS, Bear JC, Johnson GJ. The burden of genetically determined eye disease. Br J Ophthalmol. 1986;70:696-699.

[18] Krumpaszky HG, Ludtke R, Mickler A, Klauss V, Selbmann HK. Blindness incidence in Germany. A population-based study from Wurttemberg-Hohenzollern. Ophthalmologica.1999; 213:176-182.

[19] Munier A, Gunning T, Kenny D, O'Keefe M. Causes of blindness in the adult population of the Republic of Ireland. Br J Ophthalmol. 1998;82:630-633.

[20] Cotter SA, Varma R, Ying-Lai M, Azen SP, Klein R. Causes of low vision and blindness in adult Latinos: the Los Angeles Latino Eye Study. Ophthalmology. 2006;113:1574-1582. 5. Buch H, Vinding T, La Cour M, Appleyard M, Jensen GB, Nielsen NV. Prevalence and causes of visual impairment and blindness among 9980 Scandinavian adults: the Copenhagen City Eye Study. Ophthalmology. 2004;111:53-61 